\title{
Cell-Penetrating, Guanidinium-Rich Oligophosphoesters: Effective and Versatile Molecular Transporters for Drug and Probe Delivery
}

\author{
Colin J. McKinlay ${ }^{\dagger} \neq$, Robert M. Waymouth ${ }^{*}, \dagger$, and Paul A. Wender ${ }^{*}, \dagger, \ddagger$ \\ †Department of Chemistry, Stanford University, Stanford, California 94305, United States \\ ‡Department of Chemical and Systems Biology, Stanford University, Stanford, California 94305, \\ United States
}

\begin{abstract}
The design, synthesis, and biological evaluation of a new family of highly effective cellpenetrating molecular transporters, guanidinium-rich oligophosphoesters, are described. These unique transporters are synthesized in two steps, irrespective of oligomer length, by the organocatalytic ring-opening polymerization (OROP) of 5-membered cyclic phospholane monomers followed by oligomer deprotection. Varying the initiating alcohol results in a wide variety of cargo attachment strategies for releasable or nonreleasable transporter applications. Initiation of oligomerization with a fluorescent probe produces, upon deprotection, a transporterprobe conjugate that is shown to readily enter multiple cell lines in a dose-dependent manner. These new transporters are superior in cell uptake to previously studied guanidinium-rich oligocarbonates and oligoarginines, showing over 2-fold higher uptake than the former and 6-fold higher uptake than the latter. Initiation with a protected thiol gives, upon deprotection, thiolterminated transporters which can be thiol-click conjugated to a variety of probes, drugs and other cargos as exemplified by the conjugation and delivery of the model probe fluorescein-maleimide and the medicinal agent paclitaxel (PTX) into cells. Of particular significance given that drug resistance is a major cause of chemotherapy failure, the PTX-transporter conjugate, designed to evade Pgp export and release free PTX after cell entry, shows efficacy against PTX-resistant ovarian cancer cells. Collectively this study introduces a new and highly effective class of guanidinium-rich cell-penetrating transporters and methodology for their single-step conjugation to drugs and probes, and demonstrates that the resulting drug/probe-conjugates readily enter cells, outperforming previously reported guanidinium-rich oligocarbonates and peptide transporters.
\end{abstract}

\section{Graphical abstract}

\footnotetext{
“Corresponding Authors: waymouth@stanford.edu; wenderp@stanford.edu. Supporting Information

The Supporting Information is available free of charge on the ACS Publications website at DOI: 10.1021/jacs.5b13452.

Supplemental figures and information regarding materials used, instrumentation, synthetic procedures, characterization data, and biological assays. (PDF)

Notes

The authors declare no competing financial interest.
} 


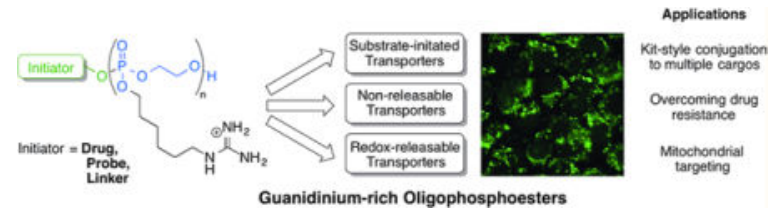

\section{INTRODUCTION}

The design and development of new strategies and agents that enable or enhance the passage of drugs and probes across biological barriers is a goal of unsurpassed significance in research, imaging, diagnostics, and therapy. ${ }^{1,2}$ Many potential drug candidates are abandoned during development simply because they do not possess the proper physical properties needed to reach their biological targets. More versatile delivery strategies would enable a broader range of therapeutic leads or diagnostic agents to be evaluated. In connection with this goal, we reported in 2000 the seminal structure-function analysis of the HIV Tat 9-mer (RKKRRQRRR), showing that the ability of this polar (oligocationic) peptide to cross nonpolar cell membranes is a function of the number and spatial array of its guanidinium groups. ${ }^{3}$ Such guanidinium-rich agents, dubbed "molecular transporters," are proposed to act as physical property "chameleons", transitioning from polar oligocations to less polar, cell-penetrating complexes as they engage cell-surface anions in electrostatic and bifurcated hydrogen-bonding interactions. ${ }^{4,5}$ Using these design criteria, we first showed that compositionally diverse guanidinium-rich scaffolds, including peptoids, ${ }^{3}$ spaced peptides, ${ }^{6}$ oligocarbamates, ${ }^{7}$ dendrimers, ${ }^{8}$ and oligocarbonates ${ }^{9}$ are able to efficiently enter cells. Noteworthy contributions from many other groups have also been reported, including guanidinium-rich modified peptides, ${ }^{10-12}$ cyclic peptides, ${ }^{13,14}$ peptide nucleic acids,,${ }^{15}$ and transporters resulting from the oligomerization of guanidinium-containing monomers such as norbornenes, ${ }^{16,17}$ methacrylamides, ${ }^{18}$ and cyclic disulfides. ${ }^{19}$ These transporters have been shown to enable or enhance the passage of numerous cargos including small molecules, ${ }^{20-22}$ peptides and proteins, ${ }^{23-26}$ and oligonucleotides, ${ }^{27-31}$ across multiple biological barriers ${ }^{1,2,32,33}$ including the cell wall of algae. ${ }^{34}$ Here, we report a new class of molecular transporters, guanidinium-rich oligophosphoesters, which exhibit increased delivery efficacy and offer several advantages over previously reported systems including our original oligoarginines and more recent oligocarbonates (Figure 1).

Synthetic polyphosphoesters have emerged in multiple areas as attractive biomaterials, ${ }^{35-37}$ and as synthetic DNA mimics. ${ }^{38-41}$ More recently, impressive contributions by Wooley and others have demonstrated that nanoparticle and micellular formulations generated from polyphosphoesters can be used in drug delivery and biomedical applications. ${ }^{42-45} \mathrm{We}$ hypothesized that the unexplored guanidinium-functionalized phosphoester backbone would address the concerns and challenges associated with previous transporter systems and result in superior delivery vehicles. First, unlike transporters accessed by solid phase techniques in which 16 synthetic steps are required to make an 8-mer due to iterative activation and coupling reactions, the oligophosphoesters can be assembled in one step using a simple organocatalytic ring-opening polymerization (OROP). ${ }^{46}$ This process avoids toxic metal contaminants associated with some metal-catalyzed oligomerizations. ${ }^{47}$ Of further 
importance, the resulting phosphotriester backbone imparts enhanced water-solubility over hydrophobic backbones such as poly(acrylates) or poly(lactic-co-glycolic acid). This suppresses oligomer aggregation in aqueous environments, especially after functionalization with hydrophobic linkers, side chains, or highly nonpolar drugs such as paclitaxel (PTX). Additionally, oligophosphoesters have increased hydrolytic stability ${ }^{48}$ over other materials obtained through anionic polymerizations, specifically carbonates and esters which are generally more hydrolytically labile $\left(t_{1 / 2}<8 \mathrm{~h}\right)$ and thus less easily stored and used. ${ }^{9}$ As a further advantage over carbon-based oligomers, phosphotriesters can uniquely accommodate side chain attachment directly at their pentavalent connecting phosphate group which allows for a much wider diversity of structure, properties and performance function. To explore these potentially advantageous transporter attributes, we set out to investigate and here report the first studies of this new class of guanidinium-rich oligophosphoester molecular transporters. We find that these transporters are superior to the commonly used oligoarginine and guanidinylated oligocarbonate delivery systems.

Guanidinium-rich oligophosphoesters obtained through OROP can accommodate multiple conjugation and drug delivery strategies depending on the functionality of the cargo drug or probe (Figure 2). For target molecules containing a nucleophilic alcohol, thiol, or amine, a cargo-initiated oligomerization can be used to directly generate the cargo-transporter conjugate (Figure 2B, Strategy 1). For other, more densely functionalized drug molecules, a postoligomerization attachment strategy can be employed (Figure 2B, Strategy 2), for which a trityl thioether initiator (5) is used to form, upon deprotection, a transporter with a free thiol linker. Conjugation can then be effected by reaction of this transporter thiol with a maleimide or iodoacetamide attached to the drug/probe for applications in which intracellular drug/probe release is not required (e.g., when a probe or drug's activity is not changed by transporter attachment or benefits from the intracellular localization of the transporter conjugate). If on the other hand release of the free drug or probe is necessary, the transporter thiol can be linked to the cargo by a disulfide bond to afford a conjugate that would release free cargo upon exposure to intracellular glutathione. ${ }^{2,49} \mathrm{~A}$ third strategy (not shown) is to complex a polyanionic cargo such as siRNA through electrostatic and hydrogen-bonding interactions as we have shown with guanidinium-rich oligocarbonates. ${ }^{28,29}$ Together, this new class of transporters and these conjugation strategies enable attachment of a wide variety of drug and probe molecules with minimal modification of the method of synthesis and delivery of probe conjugates or free probe after intracellular release.

\section{RESULTS AND DISCUSSION}

\section{Design and Synthesis of a Guanidinium-Functionalized Cyclic Phospholane Monomer for Ring-Opening Oligomerization}

A 6-carbon linker was chosen to connect guanidinium groups to the oligophosphoester backbone based on our previous optimization of side-chain length for peptoid molecular transporters. ${ }^{3}$ The use of this hydrophobic hexyl linker is enabled by the increased aqueous solubility of the phosphotriester backbone. The requisite guanidinium-containing monomer was easily prepared by condensative coupling of the corresponding Boc-protected 6- 
hydroxyhexyl guanidine 2 with 2-chloro-1,3,2-dioxaphospholane-2-oxide (COP) at $0{ }^{\circ} \mathrm{C}$ in THF using triethylamine as an $\mathrm{HCl}$ scavenger (Scheme 1). Coupling proceeded in nearly quantitative yield, though purification of the resulting monomer, as noted for other cyclic phospholanes, was challenging due to the instability of cyclic phospholanes to heat, moisture, and silica ${ }^{50}$ However, pure HexPhos monomer 3 was obtained in $79 \%$ isolated yield as a highly viscous liquid by filtration of triethylammonium salt and removal of solvent and excess COP under high vacuum, followed by trituration with diethyl ether.

\section{Oligomerization of Hexyl-guanidinium Phospholane (HexPhos) Monomer}

The synthesis of guanidinium-rich oligophosphoesters was accomplished through OROP of the strained HexPhos monomer $\mathbf{3}$ following the procedure adapted to phosphotriesters by Yamaguchi and Jérôme (Figure 2A). ${ }^{51,52}$ This strategy has been used by us and others for the synthesis of a variety of linear and cyclic polyphosphoesters. ${ }^{53,54}$ Oligomerizations were conducted under moisture-free conditions in a nitrogen-purged glovebox by dissolving HexPhos monomer 3, a primary alcohol initiator (4 or 5), and thiourea catalyst in dichloromethane, followed by addition of 1,8-diazabicycloundec-7-ene (DBU) to catalyze ring-opening. A plot of molecular weight versus conversion is consistent with the living nature of polymerization, showing a linear increase in molecular weight throughout the reaction with conversion up to $88 \%$, and consistent polydispersity $\left(M_{\mathrm{w}} / M_{\mathrm{n}}\right)$ under 1.4 (Supporting Information, Figure S1, S2).

Using this simple, one-flask procedure, oligomers of a variety of lengths were synthesized by controlling the initiator to (DP) were consistent with target monomer/initiator ratios and lengths from 8 to 25 were readily obtained, demonstrating the ability of this synthetic method to rapidly produce desired oligomers in a length-selective fashion. Boc-protected oligomers were deprotected by treatment with $10 \% \mathrm{v} / \mathrm{v}$ trifluoroacetic acid in dichloromethane to expose the requisite cationic guanidinium groups. Deprotection occurred in near quantitative yield, resulting in complete loss of Boc groups without backbone degradation as determined by ${ }^{1} \mathrm{H}$ and ${ }^{31} \mathrm{P}$ NMR. When the tritylmercaptohexanol initiator (5) was used, $10 \% \mathrm{v} / \mathrm{v}$ ethanedithiol was added after deprotection as a trityl cation scavenger and to reduce any dimers resulting from disulfide formation. All resulting cationic oligomers were freely water and PBS-soluble for use in in vitro assays.

\section{Hydrolytic Stability of HexPhos Oligomers}

New guanidinium-rich oligophosphoester transporters exhibited increased hydrolytic and biological stability relative to oligocarbonates and oligoesters. The hydrolysis of HexPhos 18 oligomer $\mathbf{6 d}$ was monitored for 25 days at $37{ }^{\circ} \mathrm{C}$ by ${ }^{31} \mathrm{P}$ NMR by comparing the relative areas of phosphotriester ${ }^{31} \mathrm{P}$ peaks $(\delta=-2$ to -3$)$, indicative of intact oligomeric units, and phosphodiester or monoester ${ }^{31} \mathrm{P}$ peaks $\left(\delta=2\right.$ to -2 ), indicating a hydrolysis product. ${ }^{50} \mathrm{In}$ acetate buffer ( $\mathrm{pH} 5.0$, consistent with late endosomes/lysosomes and skin), the oligomer showed a high degree of hydrolytic stability, with approximately $10 \%$ degradation occurring after 25 days (Supporting Information, Figure S5, S6). Under neutral conditions (HEPES buffer $\mathrm{pH} 7.4$, cytosol and bloodstream) the oligophosphoester exhibited a half-life of approximately 22 days. The fastest rates of hydrolysis occurred under basic conditions (Tris$\mathrm{HCl}$ buffer $\mathrm{pH} 9.0$, mitochondria and intestinal tract), with an approximate half-life of $\mathbf{6}$ 
days. The same pH-dependence of hydrolysis has been observed for amine-functionalized phosphotriesters, in contrast to oligomers with aliphatic or neutral side chains which show no increase in hydrolysis under basic conditions. ${ }^{45,55}$ HexPhos oligomers appeared to be inert to cleavage by Phosphotriesterase I, an enzyme previously reported to catalyze the hydrolysis of simple linear phosphoesters ${ }^{56}$ with hydrolysis rates being similar to those in neutral ( $\mathrm{pH}$ 7.4) buffer. This is advantageous for their utility in the bloodstream, GI tract, and tumor tissues where phosphatase concentrations are known to be elevated. ${ }^{57-59}$ Incubation in fetal bovine serum (FBS) afforded hydrolysis rates very similar to the neutral buffered condition, indicating that the presence of other biomolecules and serum proteins does not significantly affect backbone degradation. These hydrolysis properties demonstrate that the oligophosphoester backbone is significantly more robust than previously studied polycarbonate $\left(t_{1 / 2}=8 \mathrm{~h}\right)^{9}$ and polyester $\left(t_{1 / 2}=1-3 \mathrm{~h}\right)^{60-62}$ systems. The increased hydrolytic stability allows for ease of storage, formulation and treatment, while still maintaining biodegradability to nontoxic components over longer time periods.

\section{Cargo-Initiated Delivery of a Fluorescent Probe by HexPhos Oligomers}

The new guanidinium-rich oligophosphoesters exhibit increased efficacy as drug delivery vehicles relative to previously studied oligopeptides and oligocarbonates. To evaluate the cellular uptake of HexPhos molecular transporters, HeLa cells were treated with dansyloligomer conjugates and analyzed by flow cytometry for the fluorescence of the dansyl sulfonamide probe $\mathbf{4}$ used as an initiator. The dansyl probe $\mathbf{4}$ alone does not enter cells on its own, and noncovalent mixtures of $\mathbf{4}$ and transporter $\mathbf{7}$ do not result in any intracellular fluorescence (Supporting Information, Figure S7). Using this assay, the relative efficiency of a variety of oligomers can be quantified and compared, and applied to future cargoconjugates obtained through Strategy 1 or Strategy 2 in Figure 2. First, an oligomer length screen was performed to determine the dependence of cellular uptake on the number of guanidinium groups in an oligomer (Figure 3A), and to compare the HexPhos oligomers to the arginine homo-oligomer (Dansyl-Arg8, 8) and the oligocarbonate system reported by Cooley, et al. (Dansyl-MTC-G8, 9). ${ }^{9}$ This screen showed maximal uptake occurring with the HexPhos 10-mer $\mathbf{6 b}$, with significant uptake occurring with the 8-mer $\mathbf{6 a}$ and 12-mer $\mathbf{6 c}$. This is consistent with our previous work with oligoarginine and oligopeptoid molecular transporters which showed a parabolic relationship of length and uptake with 16-mers being optimal. ${ }^{3}$ The decrease in uptake for the HexPhos 18-mer (6d) and 25-mer (6e) may be due to increased toxicity of those compounds, since the averaged flow cytometry data includes both living and dead cells, resulting in lower fluorescence for populations containing more nonviable cells.

As illustrated in Figure 3A, the superior performance of the Dansyl-HexPhos8 relative to the Dansyl-Arg8 (8) and Dansyl-MTC-G8 (9) transporters is dramatic. HexPhos oligomers were taken into cells to higher degrees than both previously reported systems, with $>6$-fold increases in fluorescence over the peptide Dansyl-Arg8 (8) and a $>2$-fold increase over the oligocarbonate Dansyl-MTC-G8 (9). This increase in uptake can be explained by the increased linker length (6-carbons) over the oligocarbonate system (2-carbons), allowing the guanidinium groups to more effectively engage with cell surface anions (e.g., phosphates, sulfates, and carboxylates) as proposed for initiation of cell entry. ${ }^{3}$ The increased 
hydrophobic density of this linker might also allow for easier partitioning into the membrane after initial association occurs. Longer side chains on other transporter systems encountered a loss in solubility not observed with these oligophosphoester transporters. ${ }^{3}$

\section{Dose and Cell Line Dependence of Uptake of HexPhos Oligomers}

The generality of cellular uptake was explored by testing oligomer internalization in a variety of human and nonhuman cell lines (Figure 3B). The Dansyl-HexPhos8 oligomer 6a was selected for analysis because it showed robust uptake and is directly comparable in charge to previously studied Dansyl-Arg8 (8) and Dansyl-MTC-G8 (9) systems. HeLa cells (human cervical cancer) were used along with Jurkat (human T-lymphocytes), OVCAR-429 (human ovarian cancer), and 4T1 (murine breast cancer) cells. Significantly, all cell lines showed robust uptake of the HexPhos 8-mer 6a, which out-performed both the Dansyl-Arg8 (8) and Dansyl-MTC-G8 (9) positive controls. Given the number of applications reported for oligoarginine systems, including their advancement into clinical trials, ${ }^{20}$ this enhanced performance of the HexPhos system widens the breadth of potential applications for drug and probe delivery. Dansyl-HexPhos8 6a shows a linear dependence on treatment concentration in HeLa cells from 5 to $25 \mu \mathrm{M}$ concentrations (Supporting Information, Figure S8). The lower bound of this range is limited only by the detection limits of the dansyl fluorophore by flow cytometry, with much lower treatment concentrations possible.

\section{Cellular Toxicity}

HexPhos oligomers exhibit low levels of cellular toxicity in MTT viability assays.

Compound toxicity increased slightly with oligomer length, a trend that was also demonstrated in other systems such as the peptides andoligocarbonates. However, toxicity thresholds for all compounds were well above typical treatment concentrations for typical molecular therapeutics or imaging probes. $\mathrm{LD}_{50}$ 's were measured at $18,12,10,9$, and $3 \mu \mathrm{M}$ for the 8-mer (6a), 10-mer (6b), 12-mer (6c), 16-mer (6d) and 25-mer (6e), respectively (Supporting Information, Figure S9).

\section{Mechanism of Uptake}

To gather insight into the mechanism of uptake of Dansyl-HexPhos8 (6a) and compare these results to other transporter systems, several conditions previously shown to influence uptake were examined (Supporting Information, Figure S10). When cells were incubated with Dansyl-HexPhos8 in PBS where sodium ions were replaced with potassium ions, a condition known to reduce the membrane potential, ${ }^{4}$ uptake was reduced by $75 \%$. In line with our earlier studies, this result indicates that the membrane potential is required in the mechanism for uptake of the new transporters. When cells were treated with Dansyl-HexPhos8 (6a) at $4{ }^{\circ} \mathrm{C}$, a condition which attenuates endocytotic entry, there was a $25 \%$ reduction in uptake. Taken together, these results suggest mixed mechanisms of cellular entry with a nonendocytotic mechanism playing a major role. Such dual or competing mechanisms have been observed previously in single molecule experiments. ${ }^{63}$ Sodium azide inhibits ATPdependent processes which inhibits both endocytosis and neutralizes the membrane potential by disabling sodium-potassium exchange pumps. ${ }^{64}$ Treatment with $\mathrm{NaN}_{3}$ resulted in significant $(86 \%)$ reduction in uptake consistent with inhibition of multiple uptake 
mechanisms. Additional mechanistic studies will be reported separately as uptake is known to be influenced by cargo size, temperature, cell type, counterion, membrane potential, $\mathrm{pH}$ and other factors.

\section{Delivery of Thiol-Reactive Probes}

A wide scope of drug and probe molecules can be installed postoligomerization using a protected thiol initiator (5) as shown in Figure 2, Strategy 2. This "clickable" conjugation strategy allows for attachment and delivery of drugs containing reactive functionalities that are not compatible with the OROP or deprotection conditions. Many fluorescent probes, small-molecule therapeutics, and peptides are available as maleimide or iodoacetamide conjugates, and can thus be attached in situ to a thiol-functionalized oligomer immediately before treatment. To explore this strategy, deprotected thiol-initiated HexPhos 8-mer (7) was mixed with fluorescein (FL) maleimide (10) in PBS at room temperature for $2 \mathrm{~h}$ to effect a Michael addition and uptake of the resulting conjugate was determined by flow cytometry (Figure 4A). Cells treated with the FL-maleimide control 10 showed little FL fluorescence, likely only arising from reaction of nucleophiles present on the cell surface. In line with this reasoning, when the FL-maleimide conjugate was prereacted with mercaptoethanol to form control conjugate 11, fluorescence was further reduced. In striking contrast, the FL-HexPhos conjugate $\mathbf{1 2}$ showed a 160-fold increase in fluorescence over the control compounds $\mathbf{1 0}$ and 11, consistent with significant cellular uptake. Treated cells showed a complete shift in population fluorescence ( $>99 \%$ transfection) as shown in the flow cytometry histogram (Figure 4B). This demonstrates the viability of maleimide coupling or other thiol-click reactions for delivery of probes or drugs for which release is not a requirement for activity.

\section{Intracellular Localization of HexPhos Oligomers}

Confocal microscopy was used to assess the intracellular localization of HexPhos oligomers and to confirm that flow cytometry results were reflective of HexPhos localization inside cells as opposed to attached to the cellular surface. FL-HexPhos8 conjugate 12 was chosen due to the brighter fluorescein fluorophore. Images were taken at two time points to observe intracellular localization immediately following ( $t=10 \mathrm{~min}$ ), and $16 \mathrm{~h}$ after treatment (Figure 5). Z-slices through the cellular equator at both time points show a large degree of staining within the cell body, with little fluorophore adhesion to the membrane. Ten minutes following treatment, fluorescence was highly diffuse with a few bright puncta, which is consistent with above mechanistic results showing a predominantly endocytosis-independent mechanism of entry. After $16 \mathrm{~h}$ of incubation, fluorescent staining becomes much more punctate and localizes around the nucleus, which is hypothesized to be the result of mitochondrial accumulation. Co-incubation of HeLa cells with FL-HexPhos8 conjugate 12 and MitoTracker, a commercial agent designed to accumulate in mitochondria, confirmed colocalization of stained regions. Pearson's correla tion coefficient (PCC) provides a quantitative measure of the increase in colocalization levels from a low PCC of $0.18 \pm 0.09$ after $10 \mathrm{~min}$ to a relatively high PCC of $0.65 \pm 0.03$ after $16 \mathrm{~h}$ (Supporting Information, Figure S11). This supports the conclusion that oligomers enter cells by a predominantly nonendocytotic path, and later accumulate in the mitochondria. Work by Kelley and coworkers demonstrated that amphipathic guanidinium-rich oligopeptides accumulated in the mitochondria due to increased hydrophobic interactions with the inner mitochondrial 
membrane, and delocalization of charge by a mixed amphipathic scaffold which aligns with the structure of the HexPhos oligomers. ${ }^{65}$ This localization could be exploited in future work by delivering chemotherapeutic agents directly to the mitochondria for increased efficacy, ${ }^{66}$ but does not preclude cytosolic delivery and release of free drug/probe (such as through a reducible disulfide) as transporters would still spend ample time in the cytosol for release to occur.

\section{Synthesis and Delivery of a Releasable Drug-HexPhos Conjugate}

To explore the utility of the HexPhos backbone for the conjugation, delivery and release of therapeutic agents, paclitaxel (PTX)-HexPhos conjugates were synthesized and their activity against PTX-resistant ovarian cancer cells evaluated. Our prior work with cultured and primary (patient derived) drug-resistant cancer cells demonstrated that $\mathrm{Arg}_{8}$-drug conjugates overcome drug resistance putatively by avoiding Pgp-related efflux. These unprecedented results have not been addressed with oligomers accessed by OROP. ${ }^{67,68}$ Because resistance is often the major cause of chemotherapy failure, we set out to test this strategy for overcoming resistance approach is that a new drug need not be developed as the transporterdrug conjugate can overcome the problems of administration of the drug alone.

Releasable PTX conjugates can be synthesized by disulfide exchange between a thiolinitiated oligomer and an activated pyridyl disulfide drug conjugate (Figure 2, Strategy 2 and more specifically, Scheme 2). The resulting disulfide-linked drug-transporter conjugate is shelf stable and enters cells by a mechanism that evades Pgp export. Once in the cytosol the conjugate encounters high glutathione concentrations (15 mM intracellular vs $15 \mu \mathrm{M}$ extracellular) ${ }^{69}$ causing cleavage of disulfide bond, ${ }^{22}$ and subsequent drug release. PTXHexPhos conjugate 16 was synthesized as shown in Scheme 2 by disulfide exchange between a thiol-initiated oligomer $\mathbf{7}$ and $\mathrm{C}^{\prime}$-pyridyl disulfide paclitaxel (15). The 4-carbon $\mathrm{C} 2{ }^{\prime}$ ester-based linker in $\mathbf{1 5}$ was selected for this study based on previous optimization of octaarginine conjugates, but other linker designs can be used to tune the rate of drug release through intramolecular cyclization following disulfide cleavage. ${ }^{22,67}$ Following removal of excess PTX by precipitation, oligomers showed 50\% drug incorporation by NMR end group analysis. Molecular weights by MALDI show an increase in molecular weight consistent with attachment of PTX and linker to make the final conjugate 16 (Supporting Information, Figure S12). Subsequently reported concentrations are based on PTX content.

The in vitro efficacy of PTX-HexPhos conjugate $\mathbf{1 6}$ was compared to free PTX in both wildtype and PTX-resistant ovarian cancer cells engineered to have high levels of Pgp upregulation. ${ }^{70}$ OVCA-429 (wild-type) and OVCA-429T (re sistant) cells were treated with free PTX (14) or the HexPhos conjugate 16 for $20 \mathrm{~min}$ in PBS, then washed and incubated for 3 days. After this time, cellular viability was assessed using a standard MTT assay to generate an $\mathrm{EC}_{50}$ value (the concentration at which overall cellular viability was half that of untreated cells) for each condition. As expected, in wild-type OVCA-429 cells both free PTX (14) and the PTX-HexPhos conjugate 16 were highly cytotoxic (Table 2). However, in the drug-resistant OVCA-429T cells, PTX alone was unable to kill cells at any concentration up to the limit of the assay $(20 \mu \mathrm{M})$. In striking contrast, the HexPhos conjugate 16 was still able to maintain efficacy with only a modest increase in $\mathrm{EC}_{50}$ to $1.36 \mu \mathrm{M}$. To verify that the 
oligomer itself was not contributing to the cytotoxicity of the conjugate, OVCA-429T cells were also treated with the unconjugated HexPhos oligomer 7. As expected the $\mathrm{EC}_{50}$ for the oligomer alone was nearly an order of magnitude higher, with $>95 \%$ viability up to $5 \mu \mathrm{M}$ (Supporting Information, Figure S13). The efficacy of these compounds can be expressed in terms of a "resistance factor" for free PTX and the HexPhos-paclitaxel conjugate 16, defined as the $\mathrm{EC}_{50}$ in resistant cells/the $\mathrm{EC}_{50}$ in wild-type cells. PTX alone (14) succumbs readily to resistance, with a resistance factor of 400 or greater. The PTX-HexPhos conjugate 16, however, shows a resistance factor of only 5.2, demonstrating the relatively small (5-fold) increase in $\mathrm{EC}_{50}$ for resistant disease.

\section{CONCLUSIONS}

We have described the design, synthesis, and biological evaluation of a new class of guanidinium-rich oligophosphoester delivery vehicles in multiple delivery applications. These oligomeric transporters are easily prepared in any length from cyclic monomers in a single oligomerization step and subsequently deprotected in high yield to afford oligomers with low polydispersity. Drugs or probes are readily attached in one step as oligomerization initiators. Oligomer-cargo conjugates produced using this method and initiated by a fluorescent probe exhibit high levels of uptake in multiple cell lines in contrast to little or no uptake for the probe alone. This cellular uptake varies as a function of oligomer length and maximizes at an average of 10 monomer units. Significantly, cellular uptake is substantially higher than the best previously reported and widely used oligoarginine and oligocarbonate systems, among the best performers reported thus far. ${ }^{2}$ In addition to their conjugation with drug or probe initiators, these oligomers can also be used to deliver probes (e.g., fluoresceinmaleimide) and drugs (e.g., paclitaxel) through a thiol initiator using a simple, "kit-style" preparation that could be of broad use in research in which probe delivery is problematic. The resulting nonreleasable fluorescein conjugates show high levels of cell entry as demonstrated by flow cytometry and confocal microscopy. A releasable paclitaxeltransporter conjugate designed to release free paclitaxel intracellularly was shown to be more effective than paclitaxel itself and to overcome PTX-resistant ovarian cancer cells. The ability to convert a drug that is ineffective against resistant disease to one that is effective by simple conjugation to a transporter provides the basis for addressing a major unmet clinical need associated with multidrug resistance. This concept should be applicable to other drugs that are Pgp substrates and that succumb to Pgp export based resistance. These oligophosphoesters represent an especially versatile new class of easily prepared and hydrolytically stable drug delivery vehicles that are demonstrably superior to other oligomeric transporters in all comparative studies thus far and can be readily conjugated with drugs and probes as needed for a variety of clinical, preclinical, and basic research applications. Their further advance in research, diagnostics, imaging and therapeutic applications is under active investigation.

\section{Supplementary Material}

Refer to Web version on PubMed Central for supplementary material. 


\section{Acknowledgments}

Support of this work through Grants NIH-CA031841 and NIH-CA031845 from the National Institutes of Health (P.A.W.) and National Science Foundation GOALI CHE-1306730 (R.M.W) is acknowledged. Support through the Stanford Center for Molecular Analysis and Design (CMAD) is further acknowledged (C.J.M.). Flow cytometry data was collected on an instrument in the Stanford Shared FACS Facility obtained using NIH S10 Shared Instrument Grant S10RR027431-01. Confocal microscopy was collected on an instrument in the Stanford Cell Sciences Imaging Facility supported by Award Number S10RR02557401 from the National Center for Research Resources (NCRR). We gratefully acknowledge Prof. Chris Contag and Prof. Lynette Cegelski for materials, tissue culture equipment, and use of a plate reader; Prof. Jianghong Rao for use of MitoTracker; Prof. Chaitan Khosla for use of HeLa cells.

\section{References}

1. Wender PA, Cooley CB, Geihe EI. Drug Discovery Today: Technol. 2011; 9:e49.

2. Stanzl EG, Trantow BM, Vargas JR, Wender PA. Acc Chem Res. 2013; 46:2944. [PubMed: 23697862]

3. Wender PA, Mitchell DJ, Pattabiraman K, Pelkey ET, Steinman L, Rothbard JB. Proc Natl Acad Sci U S A. 2000; 97:13003. [PubMed: 11087855]

4. Rothbard JB, Jessop TC, Lewis RS, Murray BA, Wender PA. J Am Chem Soc. 2004; 126:9506. [PubMed: 15291531]

5. Mitchell, Dj, Steinman, L., Kim, Dt, Fathman, Cg, Rothbard, Jb. J Pept Res. 2000; 56:318. [PubMed: 11095185]

6. Rothbard JB, Kreider E, VanDeusen CL, Wright L, Wylie BL, Wender PA. J Med Chem. 2002; 45:3612. [PubMed: 12166934]

7. Wender PA, Rothbard JB, Jessop TC, Kreider EL, Wylie BL. J Am Chem Soc. 2002; 124:13382. [PubMed: 12418880]

8. Wender PA, Kreider E, Pelkey ET, Rothbard J, VanDeusen CL. Org Lett. 2005; 7:4815. [PubMed: 16235896]

9. Cooley CB, Trantow BM, Nederberg F, Kiesewetter MK, Hedrick JL, Waymouth RM, Wender PA. J Am Chem Soc. 2009; 131:16401. [PubMed: 19860416]

10. Rueping M, Mahajan Y, Sauer M, Seebach D. ChemBioChem. 2002; 3:257. [PubMed: 11921409]

11. Futaki S, Nakase I, Suzuki T, Zhang, Sugiura Y. Biochemistry. 2002; 41:7925. [PubMed: 12069581]

12. Fillon YA, Anderson JP, Chmielewski J. J Am Chem Soc. 2005; 127:11798. [PubMed: 16104758]

13. Lättig-Tünnemann G, Prinz M, Hoffmann D, Behlke J, Palm-Apergi C, Morano I, Herce HD, Cardoso MC. Nat Commun. 2011; 2:453. [PubMed: 21878907]

14. Qian Z, Liu T, Liu Y-Y, Briesewitz R, Barrios AM, Jhiang SM, Pei D. ACS Chem Biol. 2013; 8:423. [PubMed: 23130658]

15. Zhou P, Wang M, Du L, Fisher GW, Waggoner A, Ly DH. J Am Chem Soc. 2003; 125:6878. [PubMed: 12783535]

16. Kolonko EM, Kiessling LL. J Am Chem Soc. 2008; 130:5626. [PubMed: 18393495]

17. Tezgel AÖ, Telfer JC, Tew GN. Biomacromolecules. 2011; 12:3078. [PubMed: 21714570]

18. Treat NJ, Smith D, Teng C, Flores JD, Abel BA, York AW, Huang F, McCormick CL. ACS Macro Lett. 2012; 1:100. [PubMed: 22639734]

19. Gasparini G, Bang E-K, Molinard G, Tulumello DV, Ward S, Kelley SO, Roux A, Sakai N, Matile S. J Am Chem Soc. 2014; 136:6069. [PubMed: 24735462]

20. Rothbard JB, Garlington S, Lin Q, Kirschberg T, Kreider E, McGrane PL, Wender PA, Khavari PA. Nat Med. 2000; 6:1253. [PubMed: 11062537]

21. Luedtke NW, Carmichael P, Tor Y. J Am Chem Soc. 2003; 125:12374. [PubMed: 14531657]

22. Jones LR, Goun EA, Shinde R, Rothbard JB, Contag CH, Wender PA. J Am Chem Soc. 2006; 128:6526. [PubMed: 16704230]

23. Chen L, Wright LR, Chen C-H, Oliver SF, Wender PA, Mochly-Rosen D. Chem Biol. 2001; 8:1123. [PubMed: 11755391] 
24. Jameson KL, Mazur PK, Zehnder AM, Zhang J, Zarnegar B, Sage J, Khavari PA. Nat Med. 2013; 19:626. [PubMed: 23603816]

25. Schwarze SR, Ho A, Vocero-Akbani A, Dowdy SF. Science. 1999; 285:1569. [PubMed: 10477521]

26. Zhou H, Wu S, Joo JY, Zhu S, Han DW, Lin T, Trauger S, Bien G, Yao S, Zhu Y, Siuzdak G, Schöler HR, Duan L, Ding S. Cell Stem Cell. 2009; 4:381. [PubMed: 19398399]

27. Kumar P, Wu H, McBride JL, Jung K-E, Hee Kim M, Davidson BL, Kyung Lee S, Shankar P, Manjunath N. Nature. 2007; 448:39. [PubMed: 17572664]

28. Geihe EI, Cooley CB, Simon JR, Kiesewetter MK, Edward JA, Hickerson RP, Kaspar RL, Hedrick JL, Waymouth R M, Wender PA. Proc Natl Acad Sci U S A. 2012; 109:13171. [PubMed: 22847412]

29. Wender PA, Huttner MA, Staveness D, Vargas JR, Xu AF. Mol Pharmaceutics. 2015; 12:742.

30. Siprashvili Z, Scholl FA, Oliver SF, Adams A, Contag CH, Wender PA, Khavari PA. Hum Gene Ther. 2003; 14:1225. [PubMed: 12952594]

31. Torchilin VP, Levchenko TS, Rammohan R, Volodina N, Papahadjopoulos-Sternberg B, D’Souza GGM. Proc Natl Acad Sci U S A. 2003; 100:1972. [PubMed: 12571356]

32. Wender PA, Galliher WC, Goun EA, Jones LR, Pillow TH. Adv Drug Delivery Rev. 2008; 60:452.

33. Torchilin VP. Adv Drug Delivery Rev. 2008; 60:548.

34. Hyman JM, Geihe EI, Trantow BM, Parvin B, Wender PA. Proc Natl Acad Sci U S A. 2012; 109:13225. [PubMed: 22847404]

35. Zhao Z, Wang J, Mao H-Q, Leong KW. Adv Drug Delivery Rev. 2003; 55:483.

36. Monge S, Canniccioni B, Graillot A, Robin J-J. Biomacromolecules. 2011; 12:1973. [PubMed: 21553908]

37. Steinbach T, Wurm FR. Angew Chem, Int Ed. 2015; 54:6098.

38. Penczek S, Pretula J. Macromolecules. 1993; 26:2228.

39. Lapienis G, Penczek S. Macromolecules. 1974; 7:166.

40. Lapienis G, Penczek S, Pretula J. Macromolecules. 1983; 16:153.

41. Klosinski P, Penczek S. Macromolecules. 1983; 16:316.

42. Zhang S, Zou J, Zhang F, Elsabahy M, Felder SE, Zhu J, Pochan DJ, Wooley KL. J Am Chem Soc. 2012; 134:18467. [PubMed: 23092249]

43. Zhang F, Zhang S, Pollack SF, Li R, Gonzalez A M, Fan J, Zou J, Leininger SE, Pavía-Sanders A, Johnson R, Nelson LD, Raymond JE, Elsabahy M, Hughes DMP, Lenox MW, Gustafson TP, Wooley KL. J Am Chem Soc. 2015; 137:2056. [PubMed: 25629952]

44. Zou J, Zhang F, Zhang S, Pollack SF, Elsabahy M, Fan J, Wooley KL. Adv Healthcare Mater. 2014; 3:441.

45. Wang J, Mao H-Q, Leong KW. J Am Chem Soc. 2001; 123:9480. [PubMed: 11562246]

46. The term "Organocatalytic ring-opening polymerization" (OROP) is used here to remain consistent with terminology in the field. However, the strategy used in this work is more accurately defined as an "oligomerization" due to the lengths of materials used.

47. Kiesewetter MK, Shin EJ, Hedrick JL, Waymouth RM. Macromolecules. 2010; 43:2093.

48. Baran J, Penczek S. Macromolecules. 1995; 28:5167.

49. Wender PA, Goun EA, Jones LR, Pillow TH, Rothbard JB, Shinde R, Contag CH. Proc Natl Acad Sci U S A. 2007; 104:10340. [PubMed: 17563383]

50. Lim YH, Heo GS, Rezenom YH, Pollack S, Raymond JE, Elsabahy M, Wooley KL. Macromolecules. 2014; 47:4634. [PubMed: 25601803]

51. Iwasaki Y, Yamaguchi E. Macromolecules. 2010; 43:2664.

52. Clément B, Grignard B, Koole L, Jerome C, Lecomte P. Macromolecules. 2012; 45:4476.

53. Stukenbroeker TS, Solis-Ibarra D, Waymouth RM. Macromolecules. 2014; 47:8224.

54. Steinbach T, Schröder R, Ritz S, Wurm FR. Polym Chem. 2013; 4:4469.

55. Baran J, Kaluzynski K, Szymanski R, Penczek S. Biomacromolecules. 2004; 5:1841. [PubMed: 15360296]

56. Wang Y-C, Tang L-Y, Sun T-M, Li C-H, Xiong M-H, Wang J. Biomacromolecules. 2008; 9:388. [PubMed: 18081252] 
57. Stella VJ, Nti-Addae KW. Adv Drug Delivery Rev. 2007; 59:677.

58. Warnes TW. Gut. 1972; 13:926. [PubMed: 4566959]

59. Domar U, Hirano K, Stigbrand T. Clin Chim Acta. 1991; 203:305. [PubMed: 1777990]

60. Zhang, Z., Ortiz, O., Goyal, R., Kohn, J. Principles of Tissue Engineering. 4th. Lanza, R.Langer, R., Vacanti, J., editors. Academic Press; Boston: 2014. p. 441-473.

61. Gombotz WR, Pettit DK. Bioconjugate Chem. 1995; 6:332.

62. Lynn DM, Langer R. J Am Chem Soc. 2000; 122:10761.

63. Lee H-L, Dubikovskaya EA, Hwang H, Semyonov AN, Wang H, Jones LR, Twieg RJ, Moerner WE, Wender PA. J Am Chem Soc. 2008; 130:9364. [PubMed: 18578528]

64. Sandvig K, Olsnes S. J Biol Chem. 1982; 257:7504. [PubMed: 7085634]

65. Rin Jean S, Tulumello DV, Wisnovsky SP, Lei EK, Pereira MP, Kelley SO. ACS Chem Biol. 2014; 9:323. [PubMed: 24410267]

66. Fonseca SB, Pereira MP, Mourtada R, Gronda M, Horton KL, Hurren R, Minden MD, Schimmer AD, Kelley SO. Chem Biol. 2011; 18:445. [PubMed: 21513881]

67. Dubikovskaya EA, Thorne SH, Pillow TH, Contag CH, Wender PA. Proc Natl Acad Sci U S A. 2008; 105:12128. [PubMed: 18713866]

68. Wender PA, Galliher WC, Bhat NM, Pillow TH, Bieber MM, Teng NNH. Gynecol Oncol. 2012; 126:118. [PubMed: 22484398]

69. Saito G, Swanson JA, Lee K-D. Adv Drug Delivery Rev. 2003; 55:199.

70. Wang YC, Juric D, Francisco B, Yu RX, Duran GE, Chen GK, Chen X, Sikic BI. Genes, Chromosomes Cancer. 2006; 45:365. [PubMed: 16382445] 


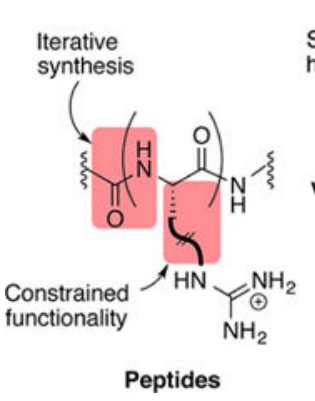

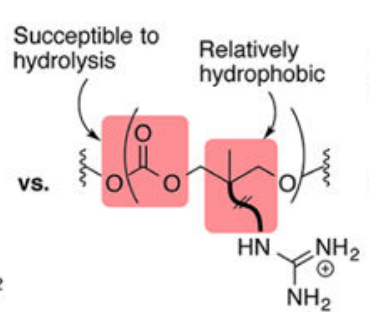

Oligocarbonates

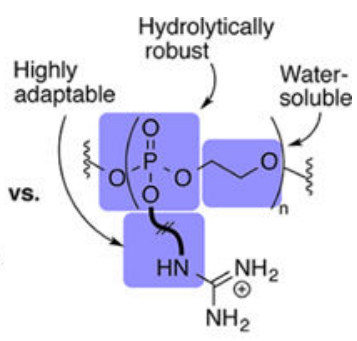

Oligophosphoesters

Figure 1.

Comparison of select oligomeric scaffolds for drug delivery to the oligophosphoesters described in this work, specifically highlighting ease of synthesis, backbone hydrophilicity, structural diversity, and aqueous stability. 
A

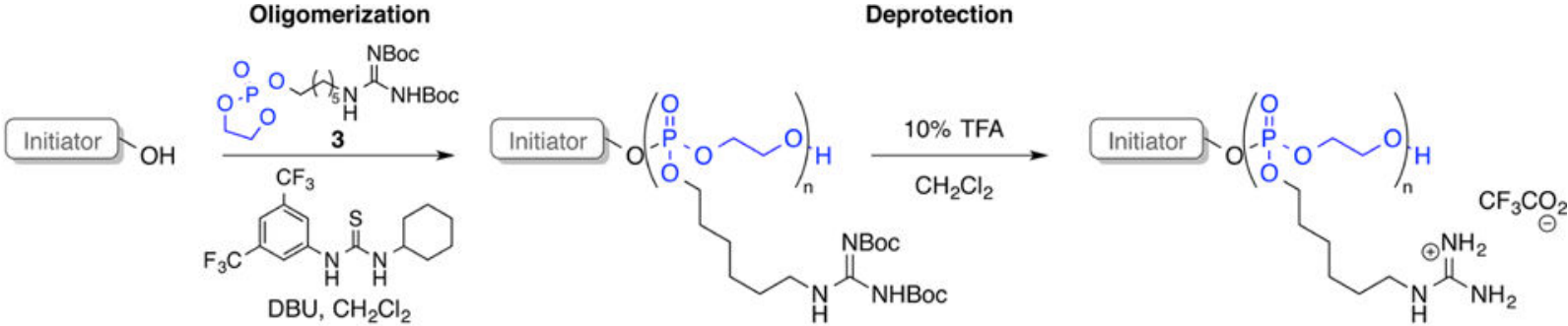

B

, $\mathrm{CH}_{2} \mathrm{Cl}_{2}$

Strategy 1:
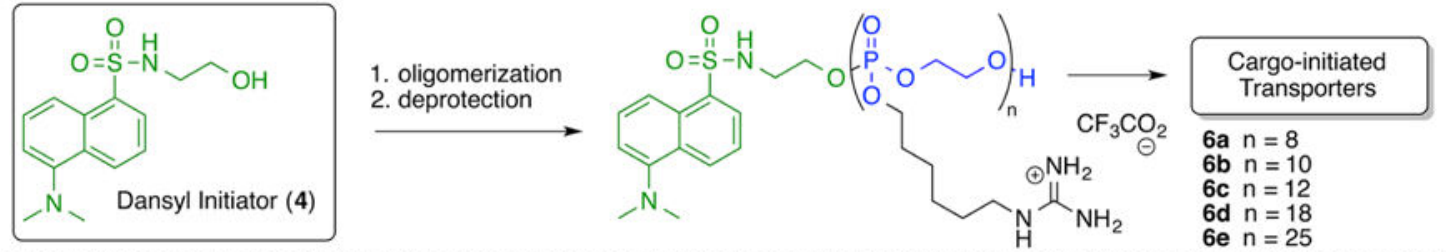

Strategy 2:

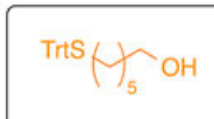

Thiol Initiator (5)

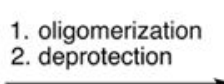
2. deprotection

Figure 2.

Overview of synthetic methodologies employed to access guanidinium-rich oligophosphoester transporters. (a) OROP of a cyclic phospholane monomer for two-step access to guanidinium-functionalized oligophosphoesters for drug/probe delivery. (b) Methods of conjugation of drugs or probe molecules to form cell-penetrating oligomeric conjugates. Strategy 1: Initiation of oligomerization by drugs or probes containing a primary alcohol, such as the dansyl initiator (4). Strategy 2: Initiation of oligomers by tritylmercaptohexanol (5) to produce, upon deprotection, oligomers containing a free thiol which can be conjugated to a variety of thiol-reactive drugs/probes, or attached through a redoxcleavable disulfide bond to form releasable drug conjugates. 

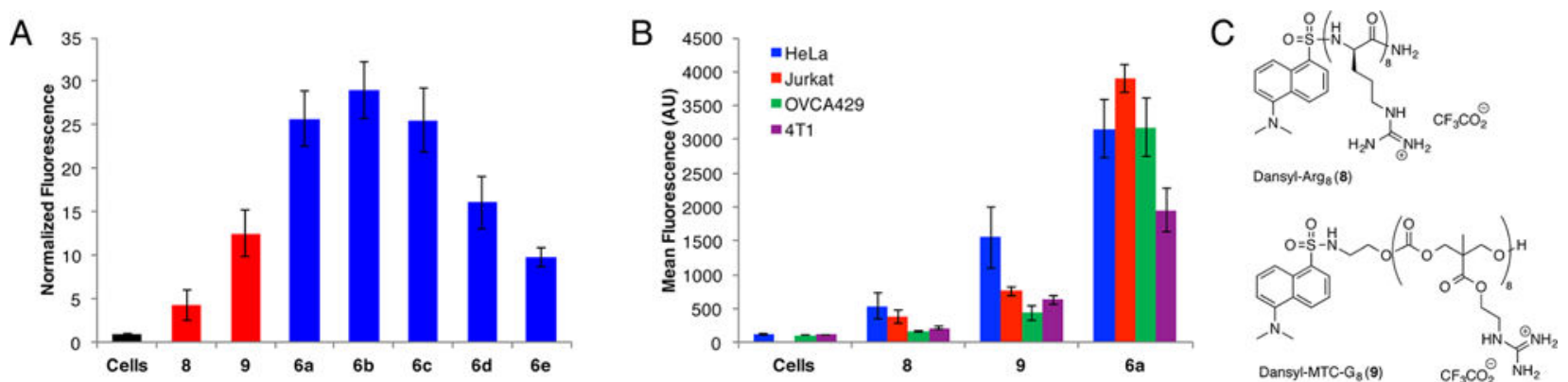

Figure 3.

Uptake of Dansyl-HexPhos oligomers compared to previously studied transporters. (a) Length dependence of uptake of Dansyl-HexPhos oligomers 6a-e in HeLa cells compared to Dansyl-Arg8 (8) and Dansyl-MTC-G8 (9). Cells were treated at $10 \mu \mathrm{M}$ for $10 \mathrm{~min}$. Fluorescence values are normalized to background fluorescence of untreated cells. (b) Cell line dependence of uptake of HexPhos8 in HeLa cells (blue), Jurkat cells (red), OVCA429 cells (green), and mouse 4T1 cells (purple). (c) Structures of previously reported transporter systems Dansyl-Arg8 (8) and Dansyl-MTC-G8 (9). 
A

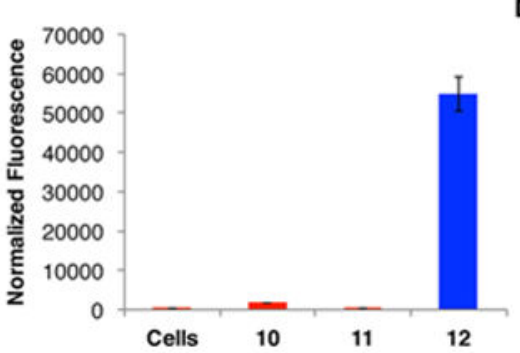

B

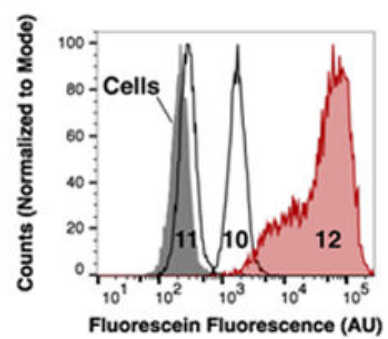

C

10

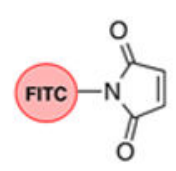

11<smiles>O=C1CC(SCCO)C(=O)N1c1ccccc1</smiles>

12

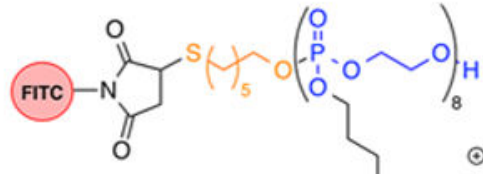

Figure 4.

(a) Uptake of FL-maleimide to HeLa cells by click-coupling to thiol-initiated HexPhos oligomer 8 determined by flow cytometry. Maleimide control and HexPhos 8 conjugate (11 and $\mathbf{1 2}$ respectively) were formed by reaction of $\mathbf{1 0}$ with the corresponding thiol. Fluorescence values are normalized to background fluorescence of untreated cells. (b) Representative flow cytometry histogram showing a complete shift in population fluorescence for cells treated with FL-HexPhos conjugate 12. (c) Structures of compounds used for FL-maleimide delivery. 


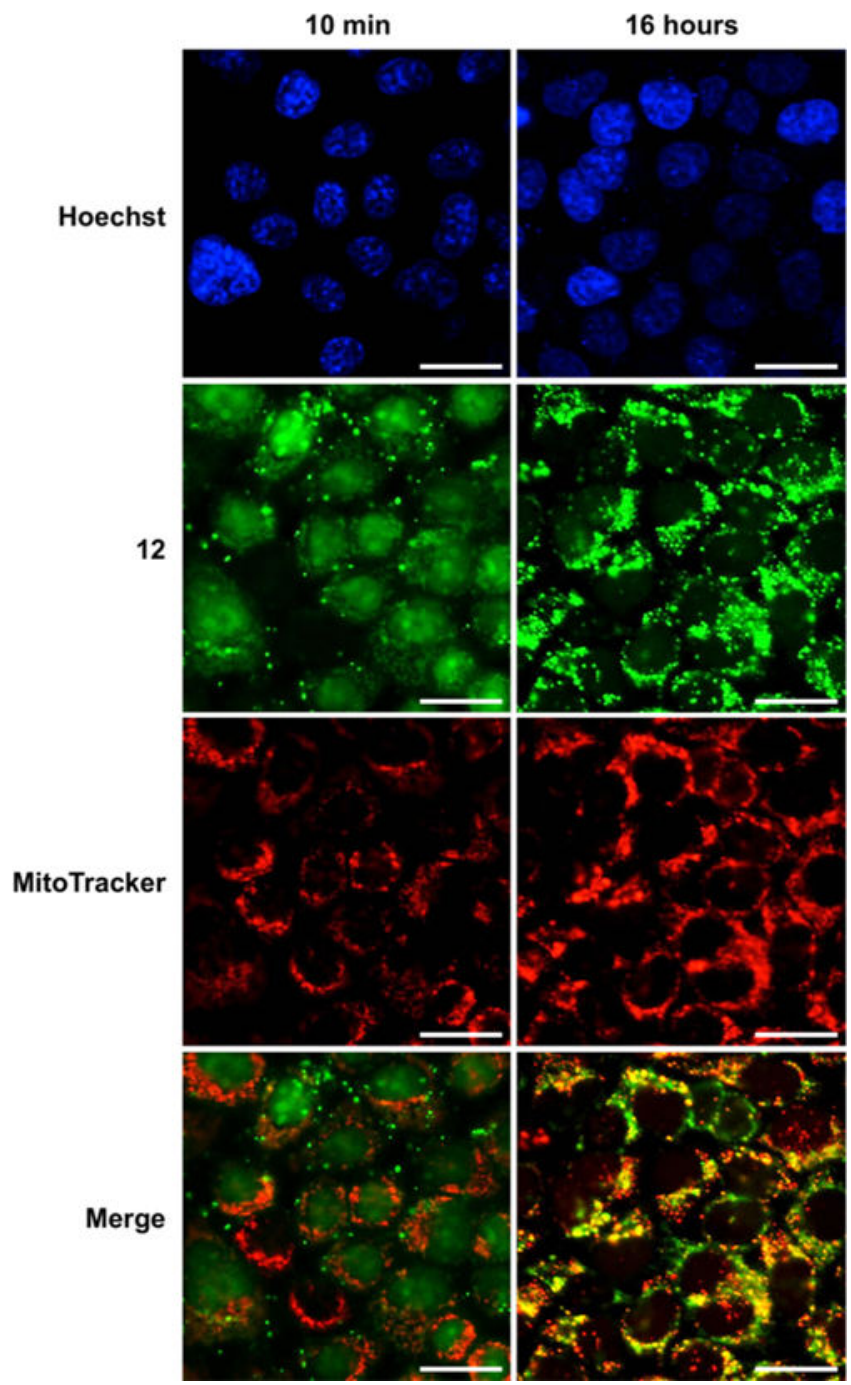

Figure 5.

Confocal microscopy images of HeLa cells treated with FL-HexPhos8 conjugate $12(10 \mu \mathrm{M})$ for $10 \mathrm{~min}$. Cell nuclei were counterstained with Hoechst 33342 and mitochondria stained with MitoTracker prior to imaging. Images were taken $10 \mathrm{~min}$ and $16 \mathrm{~h}$ following treatment. Scale bars represent $25 \mu \mathrm{m}$. 

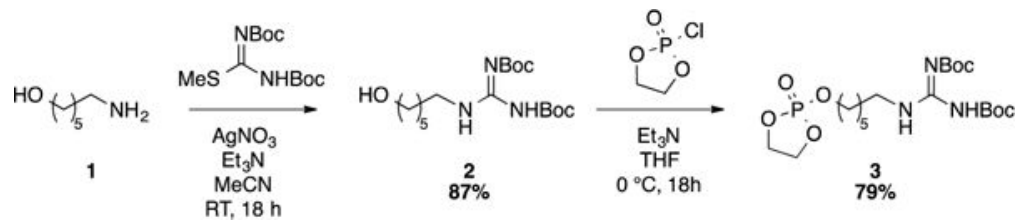

Scheme 1.

Synthesis of Protected Guanidinylated Cyclic Phospholane Monomer 2-(6-bis-Bocguanidino-hexyloxy)-1,3,2-dioxaphospholane-2-oxide (HexPhos, 3) 


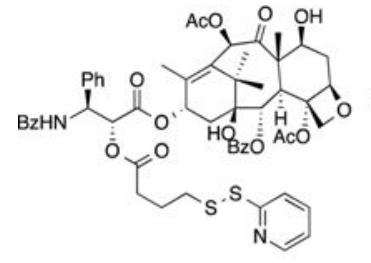

15

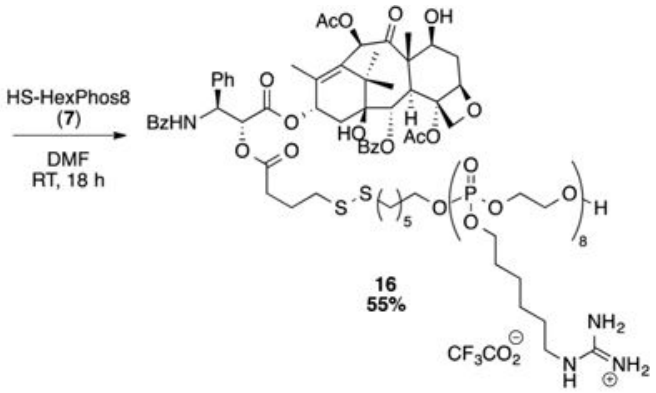

Scheme 2.

Synthesis of PTX-HexPhos Conjugate 16 by Disulfide Exchange of Activated PTXDisulfide 15 and Thiol-Initiated HexPhos Oligomer 7 
Table 1

Guanidinium-Functionalized Oligomers Synthesized by Organocatalytic Ring-Opening Oligomerization

\begin{tabular}{lllcc}
\hline entry & initiator & DP $(\mathbf{N M R})^{\boldsymbol{a}}$ & $\boldsymbol{M}_{\mathbf{n}}(\mathbf{G P C})^{\boldsymbol{b}}$ & $\boldsymbol{M}_{\mathbf{w}} / \boldsymbol{M}_{\mathbf{n}}(\mathbf{G P C})^{\boldsymbol{b}}$ \\
\hline $\mathbf{6 a}$ & Dansyl (4) & 8 & 2537 & 1.31 \\
$\mathbf{6 b}$ & Dansyl (4) & 10 & 2718 & 1.42 \\
$\mathbf{6 c}$ & Dansyl (4) & 12 & 3262 & 1.22 \\
$\mathbf{6 d}$ & Dansyl (4) & 18 & 3594 & 1.31 \\
$\mathbf{6 e}$ & Dansyl (4) & 25 & 3472 & 1.38 \\
$\mathbf{7}$ & Trityl-hexyl (5) & 8 & 2838 & 1.37 \\
${ }^{a}$ DP calculated by end group analysis. & \\
${ }_{b}$ \\
$M_{n}$ and $M_{W} / M_{n}$ determined for protected oligomers by gel permeation chromatography (GPC) in THF relative to polystyrene standards
\end{tabular}


Table 2

Cellular Toxicity $\left(\mathrm{EC}_{50}\right)$ for Free PTX and PTX-HexPhos Conjugates in Wild-Type and Resistant OVCA-429 Ovarian Cancer Cells

\begin{tabular}{|c|c|c|c|}
\hline \multirow[t]{2}{*}{ compound } & \multicolumn{2}{|c|}{$\mathrm{EC}_{50}(\mu \mathrm{M})^{a}$} & \multirow[b]{2}{*}{ resistance factor $b$} \\
\hline & OVCA-429 (wild-type) & OVCA-429T (resistant) & \\
\hline PTX Alone (14) & $0.051 \pm 0.037$ & $>20$ & $>400$ \\
\hline PTX-HexPhos8 (16) & $0.26 \pm 0.073$ & $1.4 \pm 0.45$ & 5.2 \\
\hline HS-HexPhos8 (7) & - & $13 \pm 2.9$ & - \\
\hline
\end{tabular}

